\title{
Prevalence of clinical manifestations of temporomandibular osteoarthrosis
}

\author{
BOCHIS Calin ", LAZAR Liviu ${ }^{2}$, NISTOR-CSEPPENTO Carmen², CIOARA \\ Felicia $^{2}$, PASCALAU Nicoleta ${ }^{2}$
}

Corresponding author: BOCHIS Calin, E-mail: calin bochis@yahoo.com,

\begin{abstract}
Introduction. The two compartments, disco-temporal and condilo-discal, which make up the temporomandibular joint, fulfill the functions of the stomatognathic system, namely: phonation, mastication and swallowing. The temporo-mandibular degenerative pathology is one of the 5 clinical entities given by the American Academy of Orofacial Pain in 1993. The symptoms are varied, dominated by pain, impaired joint mobility, cracks, sensation of muscle fatigue in the masseter and temporal muscles. Most cases benefit from conservative treatment, consisting of anti-allergic and anti-inflammatory medication, specific orthodontic treatment, electrotherapy (TENS, ultrasound, magnetodiaflux, laser), kinesiotherapy. 2-5\% of patients require surgery. Material and method. We performed a retrospective study, over a 9-year period (January 2010-March 2019), in which we included 503 patients who went to the Timisoara Oro-Maxillofacial Surgery Clinic, with a definite diagnosis of temporomandibular algodisfunctional syndrome, of different etiologies, between the ages of 6 and 85 years. We evaluated the etiology of temporomandibular algodisfunctional syndrome, the prevalence of clinical manifestations in temporomandibular osteoarthritis and their evolution after 10 days of conservative treatment. Results and discussions. Pain is present in $100 \%$ of cases, the values obtained in these patients with temporomandibular osteoarthritis are between 10 and $40 \mathrm{~mm}$. Cracks are present in women in the percentage of $40.8 \%$ and $37.9 \%$ in men; mobility limitation is found in women in $11 \%$ of cases, and in men in $15 \%$ of cases. Conclusions. The existence of a protocol for the diagnosis of TM osteoarthritis is essential to establish the treatment plan and prognosis of the disease. The pain from osteoarthritis TM is low intensity, present in all patients; cracks and limiting mobility are present in comparable proportions, regardless of gender. The response to treatment was optimal at 21 days of treatment.
\end{abstract}

Key words: temporomandibular osteoarthritis, clinical manifestations, prevalence,

\section{Introduction}

The two compartments, superior and inferior, which make up the temporal-mandibular joint, fulfill the functions of the stomatognathic system, namely: phonation, mastication and swallowing. Temporomandibular algodisfunctional syndrome, with intraarticular interest, can be caused by inflammatory and degenerative disorders, disorders of the articular disc displacement, modification of the articular reports, dislocations, tumors. Temporomandibular degenerative pathology is one of 5 clinical entities provided by the American Academy of Orofacial Pain in 1993. The TM joint may undergo local degenerative changes at the joint tubercle, or extend to the entire joint (1). The symptomatology is varied, dominated by pain, affecting the joint mobility, cracks, sensation of muscular fatigue in the masseter and temporal muscles (2). Extra-articular manifestations may occur, such as cervical, headache, even fibromyalgia, with psycho-social implications. Most cases benefit from conservative treatment (3), consisting of anti-allergic and anti-inflammatory medication, specific orthodontic treatment, electrotherapy (TENS, ultrasound, magnetodiaflux, laser), kinesiotherapy (4). $2-5 \%$ of patients require surgery.

\section{Material and method.}

We performed a retrospective study, over a 9-year period (January 2010-March 2019), in which we included 503 patients who went to the Timisoara Oro-Maxillofacial Surgery Clinic, with a definite diagnosis of temporomandibular algodisfunctional 
syndrome, of different etiologies, between the ages of 6 and 85 years. We evaluated the etiology of temporomandibular algodisfunctional syndrome, the prevalence of clinical manifestations in temporomandibular osteoarthritis and their evolution after 10 days of conservative treatment.

Results and discussions. The mean age of the initial batch is $41.92 \pm 16.52$. The studied group consists of 354 women, representing $70.38 \%$ of the total patients included in the study and 149 men, 29.62\%. Distribution by age: we observe an increased incidence in the age group 21-30 years, a similar incidence in the age groups 31-40 and 41-50, respectively 51-60 and 61-70, as observed in fig. 1 .

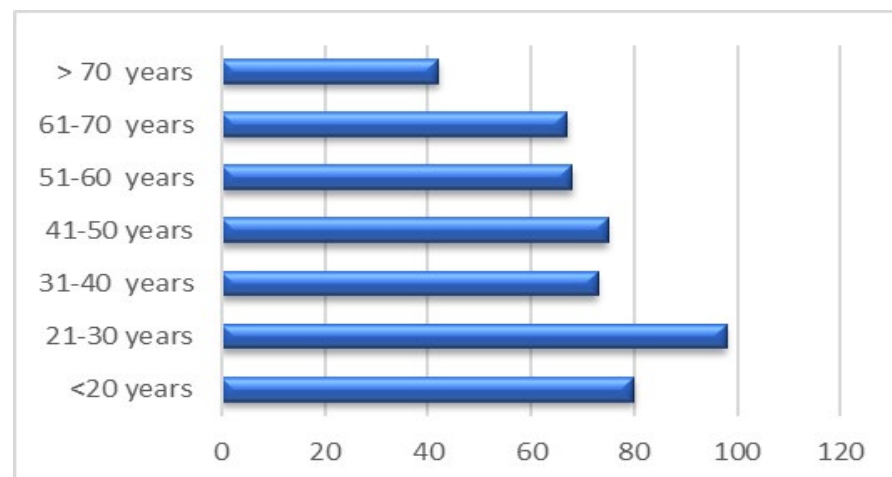

Fig.1. Distribution by age $(\mathrm{N}=503)$

Depending on the area of origin, we have a ratio of 1.7: 1 in favor of patients from urban areas. From the point of view of the clinical entities, we encountered the following distribution: 3 cases of TM arthritis, 2 cases of tumors, 2 cases of osteitis, 22 cases of post-traumatic disease, TM osteoarthritis 212 cases, unspecified etiology 262 cases. The 3 cases of TM arthritis represent the TM joint in the context of rheumatoid arthritis. Arthrosis TM represents $42 \%$, the clinical entity with the highest incidence, which characterizes the age group over 45 years (table 1).

Table 1. Distribution of cases according to etiology $(\mathrm{N}=503)$.

\begin{tabular}{|l|l|l|}
\hline Diagnosis & women & men \\
\hline $\begin{array}{l}\text { TM dysfunction (arthralgia, } \\
\text { unspecified etiology) }\end{array}$ & 169 & 93 \\
\hline Arthrosis TM & 167 & 45 \\
\hline Contusion, dislocation & 12 & 10 \\
\hline Arthritis TM & 3 & 0 \\
\hline Tumors, infections & 3 & 1 \\
\hline
\end{tabular}

Statistical processing of the incidence of clinical manifestations in patients with algodisfunctional syndrome $(\mathrm{N}=503)$ shows that pain is present in $98.61 \%$ of cases, cracks and cracks occur in $82.5 \%$ of cases, limiting mobility and deviation of jaw movements are present in $13.52 \%$ of cases.

Table 2 shows the incidence of clinical manifestations in patients with TM osteoarthritis. We find that the pain is present in $100 \%$ of cases, the values obtained in these patients with temporomandibular arthrosis are between 10 and 40 $\mathrm{mm}$, so we obtained low intensity values. Cracks are present in women in the percentage of $40.8 \%$ and $37.9 \%$ in men; mobility limitation is found in women in $11 \%$ of cases, and in men in $15 \%$ of cases. Cracking and limiting mobility are present in comparable proportions, regardless of gender.

Table 2. Distribution of clinical manifestations in osteoarthritis TM $(\mathrm{N}=212)$.

\begin{tabular}{|l|l|l|}
\hline $\begin{array}{l}\text { Clinical } \\
\text { manifestations }\end{array}$ & women & men \\
\hline Pain & $100 \%$ & $100 \%$ \\
\hline Crackles & $40.8 \%$ & $37.9 \%$ \\
\hline Limitation of mobility & $11 \%$ & $15 \%$ \\
\hline
\end{tabular}

$45 \%$ of the patients included in the study have bilateral TM joint damage. The correlation between osteoarthritis and unstable occlusion is known. Our study shows the presence of occlusion instability in $59.32 \%$ of cases.

All patients in the subtype diagnosed with osteoarthritis TM followed a conservative treatment, as appropriate, with non-steroidal anti-inflammatory, orthodontic, prosthetic, local infiltration, antiallergic electrotherapy, specific kinesiotherapy. Pain and mobility assessed at 7, 14, 21 days are shown in table 3. Favorable results are obtained after 21 days, in $61.23 \%$ of cases, $16.30 \%$ are stationary and without algal accuses and the limitation of mobility is found in $22.47 \%$ of cases.

Table 3. Clinical evolution of pain and mobility

\begin{tabular}{|l|l|l|l|}
\hline Evaluation & 7 days & 14 days & 21 days \\
\hline Improved & $34,79 \%$ & $50,89 \%$ & $61,23 \%$ \\
\hline Stationary & $65,21 \%$ & $48,51 \%$ & $16,30 \%$ \\
\hline Cured & $0 \%$ & $0,60 \%$ & $22,47 \%$ \\
\hline
\end{tabular}




\section{Discussion.}

Orofacial pain, commonly encountered, with a prevalence of $10-30 \%$ among the population (4), has rare clinical manifestations in childhood, but

2-3 times more commonly encountered in women, between the ages of 20-50 years. Studies show that only $3-7 \%$ of patients with TM disorders seek medical care (1). TM disorders can be classified into 3 groups: muscle disorders (group I), displacement of the disc (group II) and arthralgia, arthritis and osteoarthritis - group III (10). In order to establish exactly the etiology of the algodisfunctional syndrome and the therapeutic conduct, in addition to the clinical examination, paraclinical, laboratory and imaging investigations, radiographs, ultrasound or MRI are required. Osteoarthritis represents cartilage deterioration, joint space narrowing and bone production. It is the result of the action of mechanical and biological factors, (TNF $\alpha$ and other proinflammatory cytokines) (5), and its prevalence increases with the age and the action of mechanical factors, having a graphical representation similar to the Gaussian curve, with a peak in decades 5 and 6 , with a decrease after 75 years (6). There are studies performed on patients aged 73-75 years, which shows radiological changes in about $70 \%$ of the patients enrolled in the study. From a symptomatic point of view, the percentages are $9.6 \%$ in men and $18 \%$ in women over 60 years. It is estimated that about $15-20 \%$ of the world's population suffers from osteoarthritis (7). The prevalence of TM osteoarthritis varies due to variations in diagnostic criteria. It is considered that the symptomatic rate would be similar to the osteoarthritis of the other joints. Studies show that $8-16 \%$ of the population suffers from TM, uni- or bilateral osteoarthritis, with a predilection for women; in this case, the alpha polymorphism of the estrogen receptor is incriminated (8). The American Academy of Orofacial Pain classifies TM osteoarthritis in the primary, when an etiologic and secondary factor cannot be identified, when a traumatic or inflammatory factor is identified. The evolution is generally favorable, with periods of remission and exacerbation. The initial phase has a variable duration, between 2 and 4 years; pain-associated synovitis appears (13), enhanced by NSAIDs, local heat or rest, with intermittent limitations of joint mobility, sounds during mobilization. The intermediate phase is characterized by destruction joint, lasting 6 months-1 year, (9), clinically occurs spontaneous pain and mobilization, cracks and limiting mobility. In the last phase, remodeling of the facial skeleton may occur, with joint deviation, unstable occlusion, malocclusion, limiting mobility; the pain is of lower intensity, chronic, accentuated by the overload of the joint. Once the diagnosis of TM osteoarthritis is established, we will pursue the following treatment objectives: decrease of pain and inflammation, preservation or increase of joint mobility, prevention of deformities. Most cases benefit from conservative treatment. It is known that the education and counseling of the patient regarding the observance of "hygiene rules" is essential in any type of osteoarthritis. In this case we recommend a soft diet, avoiding the use of maximum angles, controlling parafunctions, use of relaxation techniques, etc., along with antiinflammatories, local topics, physical therapy, local infiltration with hyaluronic acid solutions, to reduce pain and increase mobility. Intra-articular injection of mesenchymal stem cells slows the progression of osteoarthritis TM, and chondrogenic induction in vitro could enhance therapeutic effects (11).

From our study results the increased incidence of degenerative pathology among women, similar to similar studies (12). We found an increased incidence of osteoarthritis, $40.1 \%$ of the cases diagnosed with algodisfunctional TM syndrome. International studies describe a prevalence of osteoarthritis of $24.6 \%$ (9). The discrepancy of the results can be given on the one hand by the low addressability mentioned above, of $3-7 \%$ for this type of affections; on the other hand, the lack of diagnostic algorithms (14). The main reason for which the patients of the Gold-Maxillofacial Surgery were addressed is the pain, of low intensity (10-40 $\mathrm{mm}$ ), present in $100 \%$ of the cases. The incidence of osteoarthritis TM is higher in the age group over 55 years, resulting in age and sex being considered as two risk factors (16). Bilateral involvement of the TM joint is identified in $45 \%$ of cases. Unstable occlusion is present in $59.32 \%$ of cases. All cases of osteoarthritis were treated conservatively, the best results are obtained after an evaluation after 21 days. 


\section{Conclusions.}

The existence of a protocol for the diagnosis of TM osteoarthritis is essential to establish the treatment plan and prognosis of the disease. The pain from osteoarthritis TM is low intensity, present in all patients; cracks and limiting mobility are present in comparable proportions, regardless of gender. The response to treatment was optimal at 21 days of treatment.

\section{References}

1. Shaffer SM, Brismée JM, Sizer PS, Courtney CA. Temporomandibular disorders. Part 1: anatomy and examination/diagnosis, Journal of Manual\& Manipulative Therapy. 2014 Feb; 22(1): 2-12.

2. Shaffer SM, Brismée JM, Sizer PS, Courtney CA Temporomandibular disorders. Part 2: conservative management. Journal of Manual\& Manipulative Therapy. 2014 Feb; 22(1):13-23.

3. Kalladka M, Quek S, Heir G, Eliav E, Mupparapu M, Viswanath A. Temporomandibular joint osteoarthritis: diagnosis and long-term conservative management: a topic review. J Indian Prosthodont Soc. 2014 Mar;14(1):6-15.

4. Sandu L, Tratamentul nefarmacologic fizical al durerii cronice oromandibulare Durerea, Revista asociaţei române pentru studiul durerii. 2009 ian- mar, 19(1):2-6 ISSN 12208752.

5. Tanaka E, Detamore MS, Mercuri LG. Degenerative disorders of the temporomandibular joint: etiology, diagnosis, and treatment. JDent Res. 2008;87(4):296307.

6. Bagge E, Bjelle A, Edén S, Svanborg A. Osteoarthritis in the elderly: clinical and radiological findings in 79 and 85 year olds. Ann Rheum Dis. 1991;50(8):535-539.

7. Egloff $\mathrm{C}$, Hügle $\mathrm{T}$, Valderrabano $\mathrm{V}$. Biomechanics and pathomechanisms of osteoarthritis. Swiss Med Wkly. 2012;19(142): w13583.

8. Kang SC, Lee DG, Choi JH, Kim ST, Kim YK, Ahn HJ. Association between estrogen receptor polymorphism and pain susceptibility in female temporomandibular joint osteoarthritis patients. Int J Oral Maxillofac Surg. 2007;36(5):391-394.

9. Manfredini D, Favero L, Gregorini G, Cocilovo F, Guarda-Nardini L. Natural course of temporomandibular disorders with low pain-related impairment: a 2-to-3-year followup study. J Oral Rehabil. 2013, Jun;40(6):43642.

10. Research Diagnostic Criteria for the Temporomandibular Disorders RDC/TMD. Newcastle School of Dental Sciences - 2003

11. Chen K1, Man C, Zhang B, Hu J, Zhu SS. Effect of in vitro chondrogenic differentiation of autologous mesenchymal stem cells on cartilage and subchondral cancellous bone repair in osteoarthritis of temporomandibular joint. Int J Oral Maxillofac Surg. 2013 Feb;42(2):240-8.

12. Bradbury J. Why do men and women feel and react to pain differently? Research suggests men and women may not process pain signals the same way. Lancet. 2003 Jun 14;361(9374):2052-3.

13. Kalladka M, Quek S, Moștenitor G, Eliav E, Mupparapu M. Temporomandibular Joint Osteoarthritis: Diagnosis and Long-Term Conservative Management: A Topic Review J Indian Prosthodont Soc. 2014 mar; 14 (1): 615.

14. Yount KA. Osteoarthiritis of the temporomandibular joint presents challenges in diagnosis and management. Practical pain management 3(4).

15. Rezazadeh F, Rahimi S. Evaluation of Chronic Orofacial Pain in Dental Patients; A 10 Years Retrospective Study Asian Journal of Medical and Pharmaceutical Researches, Mar 25, 2017,7(1): 01-05.

16. Kowalchuk RM, Kowalchuk RO, Kaplan-List K, Caplash JM, Block P. Temporomandibular Joint Internal Derangement Score (TIDS): novel magnetic resonance imaging assessment score and its relation to invasive treatment in patients with clinical temporomandibular joint pathology, Heliyon. 2018 Nov; 4(11): e00916. 